\title{
CALIBRATION OF HDM-4 FOR USE IN PAVEMENT MANAGEMENT SYSTEMS (PMS)
}

\author{
N. K. Mushule \\ Department of Transportation and Geotechnical Engineering \\ College of Engineering and Technology \\ University of Dar es Salaam \\ P.O.Box 35131, Dar es Salaam \\ Email:nkmmushule@yahoo.co.uk,mushule@ce.udsm.ac.tz
}

\begin{abstract}
One of major problems of road management in developing countries is the lack of decision making tools for preparing road maintenance programmes. This results in poor resource allocation and poor utilisation of existing assets. Pavement management systems (PMS) have been developed around the world in order to assist with the pavement management process in a co-ordinated and systematic manner. Some developed countries have derived pavement performance models that are suited to local conditions by setting up long term pavement performance studies. Conversely, most developing countries do not have the resources required to set up similar large-scale field experiments. However, validation and calibration of models developed from comprehensive studies elsewhere offers a viable alternative for such countries. This paper evaluates the feasibility of using HDM-4 as a support software and determines level 1 calibration factors for PMS in Tanzania. The paper demonstrates the use of a calibrated HDM-4 to determine the required road management information in developing countries.
\end{abstract}

Key word: PMS, HDM-4, calibration, strategy analysis, road network performance

\section{INTRODUCTION}

As many other developing countries, Tanzania is confronted with acute shortage of resources and genuine competing demands for the same from various development and social service programmes. Consequently, not all road sections that need maintenance or rehabilitation can be funded.

Under such budget constraints, a proper pavement management system (PMS) should be able to answer a number of questions which include: (Bhandari et al, 1987)

- Which treatments and for which road sections have the highest pay-off?

- Which road sections can be funded under the given budget constraints?

- What are the effects of various budget levels on long term road network condition and the associated agency and user costs?

- What is the optimum budget level which would keep the road network at the desired level of serviceability?

To answer these questions, the PMS must be capable of looking into the future and predicting the network condition under different maintenance strategies. In addition, not only that the PMS must be able to select cost-effective maintenance programmes but also it must have the ability to optimise maintenance expenditures under budget constraints. Essentially, these issues can be fully addressed only if the PMS incorporates a road deterioration prediction model, a road user effects model an economic evaluation and optimisation model.

\section{CRITERIA FOR MODEL SELECTION}

Several countries have developed local empirical models by setting up projects specifically for long term pavement performance study. This approach requires extensive investment in terms of time, expertise and money for the study of long term behaviour of roads with different construction 
materials in different environments and subjected to different maintenance strategies. Such a study also determines effects of pavement performance on road user costs.

An alternative approach is to extrapolate future pavement prediction from performance history. This is possible only for the agencies that have substantial amount of historical data. From previous performance trends, future road condition can be predicted.

For some agencies these two approaches are not possible simply because of lack of required resources and, at the same time, historical performance data are not available. This situation is found in most developing countries including Tanzania. Validation and calibration of models developed from comprehensive studies offers a viable alternative for such countries (Bennett and Paterson, 2000).

Existing models vary in scope, concept and approach. The selection of models to be calibrated and validated in order to suit local conditions should be done very carefully. This evaluation process, must be done in a rational and systematic way which considers factors such as: (Robinson and May, 1997)

- relevance and appropriateness of the models with regard to the agency's specific requirements;

- $\quad$ reliability in producing required outputs; and

- the affordability of the agency in satisfying current and future requirements of the selected models.

\section{USE OF HDM-4 AS A PMS SUPPORT SOFTWARE}

\section{General Background}

In a search for universally applicable performance models, a major international study was carried out which resulted into the development of a Highway Design and Maintenance Standards Model (HDM-III) (Watanatada et al, 1987). This is a well researched model with underlying performance prediction sub-models formulated using structured mechanistic-empirical approach from extensively collected data for different ranges of roadway, traffic and environmental characteristics. This approach afforded the model a great deal of transferability across a wide range of application environments. In addition, the model incorporates a full economic evaluation model with the capability of selecting alternatives that maximises benefits to the society. As a result the model has been used in over 100 different countries for planning management and economic evaluation of their highway maintenance and rehabilitation programmes (Paterson and Bennett, 1998). Furthermore, several examples of using HDM-III to support network PMS have been documented elsewhere (Mrawira and Haas, 1996).

Although HDM-III was originally developed to mostly cater for the needs of developing countries, it was observed from experience that even developed countries were using the model (Kerali et al, 1998a) In order to keep pace with the technological advancement as well as satisfying the desire of developed countries to have a reliable decision making tool in the road sector, another major study was set up. In broadening the scope of HDM-III, the study took advantage of existing knowledge without undertaking major empirical studies (Kerali et al, 1996) Based on the results of this study a Highway Development and Management Tool (HDM-4) was developed.

In this paper, the feasibility of using HDM-4 as a support software for PMS is evaluated for a case study of Tanzania.

The following criteria are used in the evaluation process:

- the ability of HDM-4 to produce outputs required from the PMS;

- $\quad$ data requirement by the software;

- $\quad$ calibration requirements;

- $\quad$ data interchange with the PMS database;

- $\quad$ upgradeability.

\section{Outputs Required}

The network level PMS for major roads in Tanzania is required to produce overall network analysis for short, medium to long term planning. This enables the Ministry of Infrastructure Development (MID) and Tanzania National Roads Agency (TANROADS) 
to come up with a rational resource allocation for financing road maintenance and rehabilitation programmes in different provinces.

HDM-4 is designed and developed to support three main levels of analysis namely project, programme and strategy analyses. Strategy and programme analyses are the most relevant application to the output required. This clearly shows that HDM-4 can be used to produce the required outputs from the network level PMS.

\section{Data Requirement}

Data acquisition is one the most expensive single items in the implementation of a PMS. Consequently, it is recommended to collect only the needed data and to collect it when needed (Smith, 1988). The amount and data detail needed depends on the level of management at which the decisions have to be made as well as the model used for analysis. For the network level analysis, aggregate data provides the desired precision. HDM-4 accepts any level of data detail from aggregate data, which may be used to define the characteristics of the road network, to most detailed data for precise definition of model parameters (Kerali et al. 1998b) A major constraint is the number of data items required by HDM-4. The software requires three groups of data. These are the data concerning road network characteristics, technical models and currency and budget. Most of road network data can be obtained from the inventory phase. However, in most cases, traffic, pavement characteristics and road condition ought to be measured for each section in the road network.

Due to the fact that modelling is not performed using aggregate data, values that translate the data into equivalent detailed data should be supplied. HDM4 supplies default values internally that can be used if the user does not supply alternative information. However, in order to achieve a reasonable precision for the intended analysis, the user should supply area specific data rather than relying on default values. Determining area-specific for each data item is so expensive that very few agencies would afford to do it. The best way of reducing data requirements for such models is to determine the most sensitive parameters that should be supplied by the user and allow the use of default values for the least sensitive parameters.
Mrawira and others investigated the possibility of using HDM-III with more default values for network level programming (Mrawira et al., 1998). They used Latin hypercube sensitivity analysis method to screen out the most sensitive link characterisation input parameters on Net Present Value (NPV) and life-cycle cost produced by HDM-III. They found the most sensitive parameters for link characterisation to be structural parameters, surface distress parameters, environment and age of pavement works. They concluded that, once these data inputs are accurately determined, other parameters can take default values without significantly affecting the resulting maintenance and rehabilitation programme. These results can be extended to link characterisation used in HDM-4 considering the similarities between the two models.

For Tanzania, climate parameters and age of pavement works should be obtained from meteorological department and MID, respectively. Structural parameters should be defined in terms of structural number derived from layer thicknesses and material properties as described previously. In order to translate aggregate data for surface distress parameters obtained from visual condition assessment, detailed measurements can be performed on sample sections rated as good, fair and poor. The results of this detailed survey from sample sections can be used as a scale on the basis of which aggregate data are translated to detailed data for the whole network.

\section{Calibration Requirements}

In the development of any model, it is not easy to consider each and every possible range of conditions. This means that not all relevant factors are considered. This was true during the development of HDMIII and, by analogy, during the development of HDM-4. Consequently, the use of HDM-4 requires calibration and validation of underlying models in order to reflect local conditions. The reliability and accuracy of the output increases with time and effort used in the model calibration and validation. Bennett and Paterson (2000) established three levels of calibration as Basic Application, Calibration and Adaptation known as levels 1, 2 and 3 respectively (Bennett and Paterson, 2000). The time required for these levels of calibration ranges from weeks for the basic level to years for the adaptation level. Basically 
two factors govern the choice of calibration level. One factor is the desired level of accuracy which depends on the type of analysis. The second factor is the affordability of the agency in terms of resources required for the selected level of calibration.

Considering the level of precision required for a network level PMS for Tanzania, as well as resource and time constraints, this research suggests that the first level of calibration should be used at the initial stage. At this level of calibration HDM-4 uses many default values and only the most sensitive parameters are locally collected. Bennett and Paterson recommend this level for general planning, quick prioritisation, preliminary screening and course estimates. In addition, they recommend traffic data, pavement characteristics, climate, unit costs and economic analysis parameters to be collected at this level (Bennett and Paterson, 2000).

At a later stage of HDM 4 use, levels 2 and 3 of calibration can be gradually introduced which will finally result into replacement of underlying relationships. However, this would require several years of commitment to local research.

\section{HDM-4 Data Interchange With PMS Database and Upgradeability}

HDM-4 consists a data import and export facility which allows the software to link with external databases with the help of a file converter.

One of the requirements of a pavement management system is the capability of being updated as new technology, more findings and up to date relationships become available. The design of HDM4 adopted a modular structure which will facilitate future modification and allow changes on underlying technical models as need arises.

\section{CALIBRATION OF HDM-4}

\section{Calibration Levels}

The use of HDM-4 requires calibration and validation of underlying relationships to reflect local conditions. As discussed earlier, there are three levels of HDM-4 calibration namely, basic application, calibration and adaptation which have been denoted as level 1, level 2 and level 3 calibration respectively.
In order to use HDM-4, it is always necessary to undertake a set-up investment of the model. This is done at the basic application level of calibration (level 1). At this level, the user adopts many default values. However, the calibration requires that basic input parameters should be provided. Subsequently, the calibration of the most sensitive parameters is considered necessary.

The study presented in this paper adopted level 1 calibration. Consequently, the calibration was done in accordance with the HDM-4 calibration reference manual (Bennett and Paterson, 2000).

\section{Sensitivity of HDM-4 Parameters}

In order to determine appropriate priority for the model calibration, the general level of sensitivity of HDM-4 to various parameters had to be determined. This helps in selecting the most important parameters, which should receive the most attention in allocating the calibration efforts. The calibration guide gives four sensitivity classes using the impact elasticity criteria. The impact elasticity has been defined as the ratio of the percentage change in a specific result to the percentage change of the input parameter while holding all other input parameters constant at a mean value. The sensitivity classes, as given by the calibration guide, are shown in Table 1. It was decided in this study that the highest calibration priority should be given to parameters with high and moderate impact elasticity.

\section{Table 1 HDM-4 Sensitivity Classes}

\begin{tabular}{|l|l|l|}
\hline IMPACT & $\begin{array}{l}\text { SENSITIVITY } \\
\text { CLASS }\end{array}$ & $\begin{array}{l}\text { IMPACT } \\
\text { ELASTICITY }\end{array}$ \\
\hline HIGH & S - I & $>0.5$ \\
\hline MODERATE & S - II & $0.20-0.50$ \\
\hline LOW & S - III & $0.05-0.20$ \\
\hline NEGLIGIBLE & S - IV & $<0.05$ \\
\hline
\end{tabular}

Calibration of Road Deterioration and Works Effects (RDWE) Relationships

\section{Calibration Parameters}

For a complete calibration of the deterioration equations, historical pavement performance data is essential. This entails the determination of pavement performance trends under known maintenance policy 
over a certain period of time. One school of thought suggests that long-term pavement performance can be estimated by taking measurements from various road sections of different ages. However, if the original pavement condition of various road sections and subsequent maintenance policies are not known, this method is not reliable. It was observed in this study that, in some cases, older pavement sections were in a relatively better condition.
In HDM-4, several defects are used to predict the deterioration of paved roads. These defects include roughness, cracking, ravelling, potholing, edge break, rutting, and surface texture.

In this study, more attention was given to parameters with high and moderate impacts. For the road deterioration and works effects, these parameters are shown in Table 2.

Table 2: Roughness environmental Parameters

\begin{tabular}{|l|l|l|l|l|l|}
\hline Temperature Category & Moisture Class & $\mathrm{m}$ & $\mathrm{km}$ & $\mathrm{m}_{\text {eff }}=\mathrm{mk}_{\mathrm{m}}$ & $\mathrm{K}_{\mathrm{ge}}=\mathrm{m}_{\text {eff }} / 0.023$ \\
\hline Tropical & Subhumid & 0.020 & 1.0 & 0.020 & 0.87 \\
\hline & Semiarid & 0.010 & 1.0 & 0.010 & 0.43 \\
\hline
\end{tabular}

Table 3: RDWE Parameters With High and Moderate Impacts

\begin{tabular}{|c|l|l|}
\hline SENSITIVITY CLASS & IMPACT ELASTICITY & PARAMETER \\
\hline S - I & $>0.5$ & $\bullet$ Structural Number ${ }^{1}$ \\
\hline & & $\bullet$ Modified Structural Number ${ }^{1}$ \\
\hline & & $\bullet$ Traffic Volume \\
\hline & $02-0.5$ & $\bullet$ Deflection ${ }^{1}$ \\
\hline S - II & & $\bullet$ Roughness \\
\hline & & $\bullet$ Annual loading \\
\hline & & $\bullet$ Age \\
\hline & & $\bullet$ All cracking area \\
\hline & & $\bullet$ Wide cracking area \\
\hline & & $\bullet$ Roughness - environment factor \\
\hline & & $\bullet$ Cracking Initiation Factor \\
\hline & & $\bullet$ Cracking Progression Factor \\
\hline
\end{tabular}

Notes: 1- Only one of the structural parameters is required, the other two are optional.

When the structural capacity is high relative to the traffic loading these parameters are in class S-II.

Source: Bennett and Paterson (2000)

In this study, all the required parameters with high impacts were determined from the field surveys (Mushule, 2002). These parameters include pavement structural number, traffic volume and roughness. In addition, for the parameters with medium impacts, annual loading and pavement age were determined from the field and MID records respectively. However, only cracking area was estimated from visual surveys.
From Table 3, the deterioration factors which need calibration are roughness- environment, cracking initiation and cracking progression. These factors have impact elasticity of $0.2,0.25$ and 0.22 respectively (Bennett and Paterson, 2000)

\section{Roughness-environment factor, Kge}

The climate in Tanzania has been classified into two climate zones. These fall within tropical temperature 
category and either subhumid or semi-arid moisture class. The calibration of the roughness environment factor, Kge, requires the determination of climate classification for both temperature category and moisture class. This factor is supposed to adjust the moisture index, $\mathrm{m}$, which has a base value of 0.023 in HDM-4. Essentially, this value signifies $2.3 \%$ annual change of roughness due to environment and is independent of traffic. The change in roughness due to environment is expressed as shown in equation 1 (Bennett and Paterson, 2000).

$$
\Delta R_{t e}=K_{g e} 0.023 R_{t}
$$

where:

$\mathrm{R}_{\mathrm{te}}=$ change in roughness component due to environment in the 1-year analysis time increment;

$\mathrm{K}_{\mathrm{ge}} \quad=$ roughness age-environment calibration factor;

$\mathrm{R}_{\mathrm{t}}=$ roughness at the beginning of the year.

For the Tanzania environment, relevant parameters presented in Table 2 were obtained using the procedure suggested by the calibration guide (Bennett and Paterson, 2000).

In this procedure the effective m-value, meff, is determined by multiplying $\mathrm{m}$ by the factor $\mathrm{km}$. This modifies the environment coefficient factor according to the road construction quality and drainage effect. In this study, it was assumed that the materials used for construction are in compliance with normal engineering standards. In addition, the drainage and formation was considered to be adequate for local conditions, and that it is moderately maintained. This assumption gives the value of $\mathrm{km}$ as 1.0 . Consequently, the obtained factor, Kge, was 0.87 and 0.43 for subhumid and semiarid moisture class respectively.

\section{Cracking initiation factor, Kci}

This factor adjusts the time it takes for the first visible crack to appear on the surface of the pavement. The factor is determined by evaluating the quality of the available refined bitumen, the likely oxidation by the atmosphere and the construction quality. For the determination of the cracking initiation factor for Tanzania, the following parameters were estimated according to the calibration guide:
- $\quad$ Low quality (LB) bitumens produced by a local refinery;

- Moderately oxidising (MO) under mixed conditions of sunshine hours and cloud cover in low to medium altitudes;

- $\quad$ Fair construction (FC) quality under moderate or variable adherence to high construction standards using 80/100 penetration bitumen. These estimate give the adjustment factor for the cracking initiation of equal to 0.8 .

\section{Cracking Progression Factor}

The calibration guide recommends that the cracking progression factor should be taken as the inverse of the cracking initiation factor. Consequently, the value of 1.25 for the cracking progression factor was assumed.

Apart from estimating adjustment factors for different calibration parameters, Bennett and Paterson (2000) point out that the only way of assessing the reliability of HDM-4 predictions is by comparing them to known local data. One of the techniques proposed is by simulating the past pavement performance.

Cracking, ravelling and potholes are characterised into two phases namely, initiation and progression phases. At the time of this study, the required data to determine most calibration factors for the progression phase were not available in Tanzania. However, the initiation calibration factors for cracking, ravelling and potholing could be evaluated with reasonable accuracy. Consequently, these factors were estimated by capturing local Tanzania experience for cracking, ravelling and potholing initiation time for new asphalt pavement roads. In this study, an estimate of time it takes for these types of distresses to initiate on a new asphalt pavement road was compared to the time predicted by HDM-4.

HDM-4 predicts the initiation of all structural cracks by the following formula:

$$
I C A=K_{\text {cia }}\left[C D S^{2} a_{0} \exp \left[a_{1} S N P+a_{2}\left(\frac{Y E 4}{S N P^{2}}\right)\right]+C R T\right]
$$

where:

ICA = time to initiation of all structural cracks (years); 


$\begin{array}{ll}\text { CDS }= & \begin{array}{l}\text { construction defects indicator for } \\ \text { bituminous surfacing; }\end{array} \\ \text { YE4 }= & \begin{array}{l}\text { annual number of equivalent standard } \\ \text { axles }(\text { million/lane }) ;\end{array} \\ \text { SNP }=\quad \begin{array}{l}\text { average annual adjusted structural } \\ \text { number of the pavement; } \\ \text { cracking retardation time due to } \\ \text { maintenance }\end{array}\end{array}$

The model predicts the cracking initiation time of 5 years for asphalt mix on granular base (AMGB) roads with no maintenance. Using the obtained adjustment factor of 0.8 the model gives the time for the first crack to appear as 4 years. However, experience shows that, with traffic loading regimes found in the country, the time for the initiation of all cracks for such a pavement ranges between $5-8$ years. 7 years were identified as the most common cracking initiation time. Consequently, it was decided to adjust the cracking initiation prediction to 7 years. In equation 2, the parameter to be adjusted is Kcia. A value of 1.4 was therefore adopted.

\section{Ravelling Initiation}

In HDM-4, the ravelling initiation time is predicted by:

$$
I R V=K_{v i} C D^{2} a_{0} R R F \exp \left(a_{1}, Y A X\right)
$$

where:

$$
\begin{aligned}
& \text { IRV = time to ravelling initiation (years); } \\
& \mathrm{CDS}=\text { construction defect indicator for } \\
& \text { bituminous surfacing; } \\
& \text { YAX }=\text { annual number of axles of all } \\
& \text { motorised vehicle types in the } \\
& \text { analysis year (million/lane); } \\
& \mathrm{Kvi}=\text { calibration factor for ravelling } \\
& \text { initiation; } \\
& \mathrm{RRF}=\text { ravelling retardation factor due to } \\
& \text { maintenance. }
\end{aligned}
$$

HDM-4 assumes that new asphalt pavements do not ravel. In this particular case, the model predicts 90 years for a new asphalt mix on granular base (AMGB) road in Tanzania to start ravelling. However, local experience from the Ministry of Infrastructure Development (MID) field personnel suggests that asphalt mix on granular base (AMGB) roads start ravelling after approximately 10 years. Consequently, in equation 2 , Kvi was adjusted to 0.11 instead of a default value of 1.0. Nevertheless, it must be pointed out that the ravelling initiation factor has been found to have negligible impacts on the pavement performance prediction.

Using these two adjusted calibration factors and keeping default values for the rest, the model predicts the initiation of wide structural cracks and pothole initiation time of 9 and 12 years respectively. These figures reasonably reflect the observed local conditions for Tanzania.

\section{Calibration of Road User Effects (RUE) Relationships}

The sensitivity of HDM-4 to various parameters affecting the Road User Effects (RUE) was also determined. The data used for the analysis were varied in order to cover a full range of conditions where the model will be applied. Finally, the variables were assigned to different sensitivity classes. Variables with high and moderate impacts are shown in Table 4.

\section{Table 4: RUE Parameters with High and Moderate} Impacts

\begin{tabular}{|l|l|l|}
\hline $\begin{array}{l}\text { SENSITIVITY } \\
\text { CLASS }\end{array}$ & $\begin{array}{l}\text { IMPACT } \\
\text { ELASTICITY }\end{array}$ & PARAMETER \\
\hline $\mathrm{S}-\mathrm{I}$ & $>0.5$ & $\begin{array}{l}\text { Replacement vehicle } \\
\text { price }\end{array}$ \\
\hline & & Parts model exponent $\mathrm{k}_{\mathrm{p}}$ \\
\hline $\mathrm{S}$ - II & $0.2-0.5$ & Roughness in IRI \\
\hline & & Parts model exponent a1 \\
\hline
\end{tabular}

Source: Bennett and Paterson, 2000.

It has been found that the road user effects (RUE) are mainly influenced by vehicle speeds and road surface roughness. As pointed out by Bennett and Paterson (2000), whilst fuel consumption is very sensitive to vehicle speeds, parts consumption is highly influenced by roughness. However, it has been shown that roughness also affects vehicle speeds. This suggests that roughness is the most important parameter that affects the road user effects. Consequently, the study reported in this paper determined roughness values from the field. In addition, the data on the replacement vehicle price were collected from the car dealers and transport operators in Tanzania.

Furthermore, for level 1 RUE calibration, most 
variables assigned the highest priority by the calibration guide were locally determined and are used as input variables to HDM-4. These variables include average mass of the vehicle, vehicle damage factor, average service life and annual vehicle utilisation.

Vehicle Fleet Characteristics used are shown in Table 5 .

\section{TYPICAL APPLICATION OF THE}

\section{CALIBRATED SOFTWARE}

To demonstrate the use of the calibrated software, a long term plan for a typical trunk road sub-network in Tanzania was determined by performing strategy analysis. Representative sections were determined by grouping road sections with similar characteristics. These sections are presented in Tables 6 and 7 for paved roads and unpaved roads respectively.

Table 5: Vehicle Characteristics for Tanzania Vehicle Fleet (2008)

\begin{tabular}{|c|c|c|c|c|c|c|c|c|}
\hline \multirow[t]{2}{*}{ Attribute } & \multicolumn{8}{|c|}{ Vehicle Name/Type } \\
\hline & $\mathrm{PC}$ & 4WD & LT & MT & HT & $\mathrm{AT}$ & $\mathrm{M} / \mathrm{Bus}$ & Bus \\
\hline No. of Wheels & 4 & 4 & 4 & 6 & 10 & 18 & 6 & 6 \\
\hline No. of Axles & 2 & 2 & 2 & 2 & 3 & 5 & 2 & 2 \\
\hline Utilisation (km/yr) & 23000 & 39000 & 39000 & 145400 & 90000 & 86000 & 30000 & 250000 \\
\hline Hours driven/yr & 550 & 1340 & 1340 & 1728 & 1920 & 2050 & 3200 & 2880 \\
\hline Average life (yrs) & 10 & 6 & 6 & 5 & 8 & 14 & 8 & 5 \\
\hline No. of Passengers & 5 & 9 & & & & & 30 & 60 \\
\hline Loading (ESAL) & 0.00 & 0.013 & 0.130 & 2.930 & 12.51 & 13.30 & 0.09 & 6.27 \\
\hline Weight (tonnes) & 1.2 & 1.8 & 3.5 & 8.0 & 10.0 & 40.0 & 3.5 & 6.0 \\
\hline Economic cost & \multicolumn{8}{|c|}{ All Costs Expressed in Tanzanian Shillings } \\
\hline - Vehicle Replac & $12 \mathrm{~m}$ & $18 \mathrm{~m}$ & $19 \mathrm{~m}$ & $25 \mathrm{~m}$ & $28 \mathrm{~m}$ & $80 \mathrm{~m}$ & $12 \mathrm{~m}$ & $60 \mathrm{~m}$ \\
\hline - Tyre cost & 40000 & 60000 & 60000 & 100000 & 100000 & 200000 & 60000 & 120000 \\
\hline - Fuel & 1000 & 1000 & 900 & 900 & 900 & 900 & 900 & 900 \\
\hline - Mtce. Labour/hr & 2000 & 2000 & 2000 & 2000 & 2000 & 2000 & 2000 & 2000 \\
\hline - Crew wages/hr & 2000 & 2000 & 2000 & 2000 & 2000 & 2000 & 2000 & 2000 \\
\hline Financial cost & \multicolumn{8}{|c|}{ All Costs Expressed in Tanzanian Shillings } \\
\hline - Vehicle Replac & $24 \mathrm{~m}$ & $36 \mathrm{~m}$ & $25 \mathrm{~m}$ & $28 \mathrm{~m}$ & $30 \mathrm{~m}$ & $120 \mathrm{~m}$ & $32 \mathrm{~m}$ & $90 \mathrm{~m}$ \\
\hline - Tyre cost & 48000 & 72000 & 72000 & 120000 & 120000 & 240000 & 72000 & 150000 \\
\hline • Fuel & 1500 & 1500 & 1500 & 1500 & 1500 & 1500 & 1500 & 1500 \\
\hline - Mtce. Labour/hr & 3000 & 3000 & 3000 & 3000 & 3000 & 3000 & 3000 & 3000 \\
\hline - Crew wages/hr & 3000 & 3000 & 3000 & 3000 & 3000 & 3000 & 3000 & 3000 \\
\hline
\end{tabular}

\section{PC- Passenger Car, LT - Light Truck, MT - Medium Truck}

\section{HT- Heavy Truck, AT- Articulated Truck}

Mtce - Maintenance, Replac - Replacement, 12m - 12 million 
Table 6: Representative Sections for Paved Roads

\begin{tabular}{|l|l|l|l|l|l|l|l|l|}
\hline $\begin{array}{l}\text { Rep. } \\
\text { Section } \\
\text { No. }\end{array}$ & Type & Roughness & Surface & Edge & Structure & Traffic & $\begin{array}{l}\text { Topo- } \\
\text { graphy }\end{array}$ & $\begin{array}{l}\text { Length } \\
(\mathrm{km})\end{array}$ \\
\hline P1 & AC & Good & Good & Good & Adequate & Low & Hilly & 33.0 \\
\hline P2 & AC & Good & Good & Good & Adequate & Low & Rolling & 126.1 \\
\hline P3 & AC & Good & Poor & Fair & Adequate & Low & Hilly & 40.3 \\
\hline P4 & AC & Fair & Good & Good & Adequate & Medium & Hilly & 16.4 \\
\hline P5 & AC & Fair & Good & Good & Adequate & Medium & Rolling & 31.5 \\
\hline P6 & AC & Fair & Good & Good & Adequate & High & Rolling & 29.4 \\
\hline P7 & SD & Fair & Fair & Fair & Critical & Low & Rolling & 35.0 \\
\hline P8 & SD & Fair & Poor & Good & Adequate & Low & Rolling & 15.0 \\
\hline P9 & SD & Fair & Poor & Poor & Adequate & Low & Rolling & 2.0 \\
\hline P10 & SD & Fair & Poor & Fair & Critical & Low & Hilly & 68.9 \\
\hline P11 & SD & Poor & Poor & Poor & Inadequate & Low & Rolling & 13.5 \\
\hline
\end{tabular}

\section{AC - Asphalt Concrete, SD - Surface Dressing}

Table 7: Representative Sections for Unpaved Roads

\begin{tabular}{|c|c|c|c|c|c|c|c|}
\hline Rep. Section No. & Roughness & Surface & Passability & Layer Strength & Traffic & Topography & Length $(\mathrm{km})$ \\
\hline U1 & Poor & Poor & Good & Low & Low & Rolling & 69.3 \\
\hline U2 & Poor & Poor & Good & High & High & Rolling & 5.9 \\
\hline U3 & Poor & Poor & Fair & Low & Low & Rolling & 29.3 \\
\hline U4 & Poor & Poor & Fair & Low & Low & Hilly & 41.7 \\
\hline
\end{tabular}

The results of the analysis are presented in Table 8. This table presents the budget forecast and the expected road network performance for paved and unpaved roads in terms of average roughness (weighted by length). This is the optimum strategic plan which would give the maximum benefits for both road agency and road users in terms of Net Present Value (NPV).

\section{Table 8: Budget Forecast and Pavement Performance Prediction}

\begin{tabular}{|l|l|l|l|l|}
\hline Year & \multicolumn{2}{|c|}{ Paved Roads } & \multicolumn{2}{c|}{ Unpaved Roads } \\
\hline & $\begin{array}{l}\text { Required Budget [million } \\
\text { TAS] }\end{array}$ & $\begin{array}{l}\text { Average Condition IRI } \\
\text { m/km }\end{array}$ & $\begin{array}{l}\text { Required Budget [million } \\
\text { TAS] }\end{array}$ & Average Condition IRI m/km \\
\hline 1 & $4,200.25$ & 3.85 & 962.7 & 17.5 \\
\hline 2 & 0 & 3.80 & 63.36 & 9.08 \\
\hline 3 & 1352.75 & 4.16 & 63.36 & 11.43 \\
\hline 4 & 577.5 & 4.31 & 63.36 & 13.46 \\
\hline 5 & 0 & 4.61 & 52.74 & 15.52 \\
\hline 6 & 9409.75 & 4.91 & $3,247.79$ & 14.19 \\
\hline 7 & 2184.00 & 4.61 & 263.16 & 14.98 \\
\hline 8 & $2,206.75$ & 4.43 & $1,011.23$ & 13.55 \\
\hline 9 & $1,352.75$ & 4.63 & 263.16 & 11.48 \\
\hline 10 & 577.50 & 4.66 & 252.54 & 14.67 \\
\hline TOTAL & $21,663.25$ & & $6,179.41$ & \\
\hline
\end{tabular}


However, the required budget is normally not available. Consequently, this study determined the effect of various budget scenarios on the performance of the road network. This has been illustrated by the determination of the paved road network performance as a function of the available budget, as shown in Table 8. The table shows clearly that the average roughness (weighted by length) improves as the budget level increases. In addition, for illustration purposes, a scenario of having $50 \%$ of the required budget for the first five years of the analysis period and $100 \%$ of the required budget thereafter was also analysed. This means that some road works must be deferred until the required budget is available. This scenario, denoted as 50+100 in Table 9, results into the average roughness of $4.76 \mathrm{~mm} / \mathrm{km}$ IRI which is better that all other scenarios except for the one with $100 \%$ of the required budget throughout the analysis period.

Table 9 Paved Road Network Performance as a Function of Available Budget

\begin{tabular}{|l|l|l|l|l|l|l|l|l|}
\hline Available Budget (\% Required Budget) & 0 & 10 & 25 & 40 & 75 & 90 & 100 & $50+100$ \\
\hline Weighted Average Roughness m/km IRI & 10.91 & 8.15 & 7.57 & 6.36 & 5.76 & 5.32 & 4.68 & 4.76 \\
\hline
\end{tabular}

\section{CONCLUSIONS}

This paper demonstrates the practicality of using HDM-4 as PMS support software. In this case, level 1 HDM-4 calibration factors for Tanzania have been suggested. These factors have been used to calibrate the software which was later used to demonstrate the determination of long term strategic planning. This was obtained in terms of budget requirements and pavement performance forecast considering the maximum benefits to both road agency and road users. In addition, the paper demonstrates that the effect of the reduced budget can also be predicted. A very important feature of HDM-4 is the provision of the facility to import and export data from and to external data source. With proper implementation of data transformation rules, HDM-4 accepts data imported from external database. This enables the user of the software to utilise the available data from the existing information system, database or road maintenance management system. However, it should be stressed that level 1 calibration of the software should be initially utilised with the provision of updating the calibration factors, for levels 2 and 3 calibration, when new information becomes available from extended long term research.

\section{REFERENCES}

Bennett, C.R. and Paterson, W.D.O., A guide to Calibration and Adaptation, Volume 5 of the Highway and Management Series, International Study of Highway Development and Management (ISOHDM), World Roads Association, PIARC, Paris, 2000.

Bhandari, A., Harral, C., Holland, E. and Faiz, A., Technical Options for Road Maintenance in Developing Countries and the Economic Consequences", In Transportation Research Record No. 1128, TRB, National Research Council, Washington D.C., pp 18 - 27, 1987.

Kerali, H.R., Odoki, J. B., and Wightman, D.C., The HDM-4 Analytical Framework, Joint 18th ARRB Transport Research Conference and Transit New Zealand Transport Symposium, Christchurch, New Zealand, pp 17-32, 1996.

Kerali, H.R., Odoki, J.B., Wightman, D.C. and Stannard, E.E., Structure of the New Highway Development and Management Tools HDM4, Fourth International Conference on Managing Pavements, Vol. 2: Implementation of Pavement Management Outputs, Durban, South Africa, pp 961 - 973, 1998a. 
Kerali, H.R., Robinson, R. and Paterson W.D.O., Role of HDM-4 in Highway Management", Fourth International Conference on Managing Pavements, Vol. 2: Implementation of Pavement Management Outputs, Durban, South Africa, pp $801-814,1998$ b

Mrawira, D. and Haas, R., Calibration of the TRRL's Vehicle-Mounted Bump Integrator, IET Journal Vol. 5, Number 5, pp 13 - 24, 1996.

Mrawira, D., Haas, R., and Paterson, W.D.O., Streamlining the World Bank's HDM III Model for Network Level Application", Fourth International Conference on Managing Pavements, Vol. 2: Implementation of Pavement Management Outputs, Durban, South Africa, pp 719 - 736, 1998.

Mushule, N.K.M., Sustainable Data Acquisition Methods for Road Maintenance Management in Developing Countries, in Uhandisi Journal, Volume 25, No. 2, Uhandisi Publications, UDSM Tanzania, pp 40 - 54, 2002.
Paterson, W.D.O and Bennett, C.R., Guidelines on Applying and Calibrating the HDM Model to Local Condition, Fourth International Conference on Managing Pavements, Vol. 2: Implementation of Pavement Management Outputs, Durban, South Africa, pp 665 - 680, 1998

Smith, R.E., A Comprehensive Ranking System for Local Agency Pavement Management, In Transportation Research Record No. 1123, TRB, National Research Council, WashingtonD.C,1988.

Robinson, R. and May, P.H., Road Management Systems: Guidelines for Their Specifications and Selection, Proceedings, Institution of Civil Engineers, Transp; 123, pp 9 - 16, 1997.Watanatada, T., Harral, CPaterson, W.D.O., Dhareshwar, A.M., Bhandari, A., and Tsunokawa, K., The Highway Design and Maintenance Standards Model, Vol. 1: Description of the HDM-III Model, John Hopkins University Press, Baltimore, 1987. 